
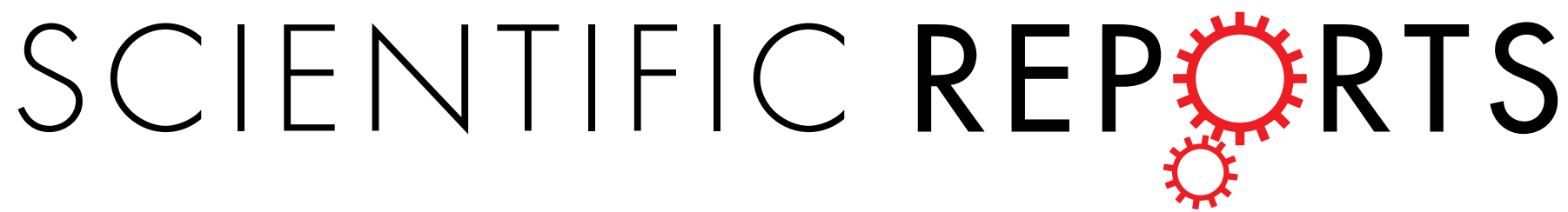

\title{
Distinct Differences on Neointima
} Formation in Immunodeficient and Humanized Mice after Carotid or

Received: 03 September 2015

Accepted: 28 September 2016

Published: 19 October 2016 Femoral Arterial Injury

Jill Moser ${ }^{1,+}$, Joris van Ark ${ }^{1}$, Marcory C. van Dijk ${ }^{2}$, Dale L. Greiner ${ }^{3}$, Leonard D. Shultz ${ }^{4}$, Harry van Goor $^{1}$ \& Jan-Luuk Hillebrands ${ }^{1}$

Percutaneous coronary intervention is widely adopted to treat patients with coronary artery disease. However, restenosis remains an unsolved clinical problem after vascular interventions. The role of the systemic and local immune response in the development of restenosis is not fully understood. Hence, the aim of the current study was to investigate the role of the human immune system on subsequent neointima formation elicited by vascular injury in a humanized mouse model. Immunodeficient NOD. Cg-Prkdc $c^{\text {scid }} / L 2$ rg $^{\text {tm } 1 W_{j l}(}$ (NSG) mice were reconstituted with human (h)PBMCs immediately after both carotid wire and femoral cuff injury were induced in order to identify how differences in the severity of injury influenced endothelial regeneration, neointima formation, and homing of human inflammatory and progenitor cells. In contrast to non-reconstituted mice, hPBMC reconstitution reduced neointima formation after femoral cuff injury whereas hPBMCs promoted neointima formation after carotid wire injury 4 weeks after induction of injury. Neointimal endothelium and smooth muscle cells in the injured arteries were of mouse origin. Our results indicate that the immune system may differentially respond to arterial injury depending on the severity of injury, which may also be influenced by the intrinsic properties of the arteries themselves, resulting in either minimal or aggravated neointima formation.

Percutaneous coronary interventions (PCIs) are widely used to treat patients with severe coronary atherosclerotic disease. Although PCI is considered an effective and safe treatment modality to establish coronary revascularization, an important drawback is the development of restenosis of the treated artery ${ }^{1}$. Restenosis is characterized by re-narrowing of the artery due to the formation of an occlusive neointima. Neointimal lesions are characterized by smooth muscle cell (SMC) proliferation and extracellular matrix deposition ${ }^{2}$. Although the causative mechanisms underlying development of restenosis are not fully understood, systemic and local inflammation are considered important driving forces ${ }^{1-3}$. Both clinical and experimental data suggest that $\mathrm{T}$ cell recruitment into the treated arterial wall promotes neointima formation, however, contradictory results have also been reported. Immunodeficiency in experimental models was found to augment neointima formation after arterial injury in both immunodeficient mice and $\mathrm{T}$ cell-depleted or athymic rats ${ }^{4-7}$. In contrast, adoptive $\mathrm{T}$ cell transfer into immunodeficient Rag $1^{\text {null }}$ mice reduced neointima formation; an effect which may be attributed to IFN $\gamma^{8}$. More recent experimental evidence identified a role for $\mathrm{CD}^{+} \mathrm{T}$ cells in inhibiting neointima formation ${ }^{7}$. However, in contrast to the protective role of $\mathrm{T}$ cells and IFN $\gamma$ on neointima formation, transfer of $\mathrm{CD} 4^{+} \mathrm{T}$ cells increased atherosclerosis in ApoE/immune deficient mice ${ }^{9}$, and IFN $\gamma$ was shown to augment native atherosclerosis ${ }^{10}$ and graft atherosclerosis ${ }^{11}$. In addition to the role of T cells in the development of restenosis, the origin of SMCs within neointimal lesions is also under debate. Although it was generally believed that neointimal SMCs are derived from the adjacent tunica media similar to SMCs in atherosclerotic lesions ${ }^{12}$, in the last 15 years it has

${ }^{1}$ Department of Pathology and Medical Biology-Pathology, University of Groningen, University Medical Center Groningen, Groningen, The Netherlands. '2Department of Pathology, Rijnstate Hospital, Arnhem, The Netherlands. ${ }^{3}$ Diabetes Center of Excellence, Program in Molecular Medicine, University of Massachusetts Medical School, Worcester, MA, USA. ${ }^{4}$ The Jackson Laboratory, Bar Harbor, ME, USA. 'Present address: Department of Critical Care and Department of Pathology and Medical Biology- Medical Biology, University of Groningen, University Medical Center Groningen, Groningen, The Netherlands. Correspondence and requests for materials should be addressed to J.-L.H. (email: j.l.hillebrands@umcg.nl) 
become evident that alpha-smooth muscle actin ( $\alpha$ SMA) expressing neointimal cells derived from circulating progenitors can also contribute to vascular remodeling ${ }^{13,14}$. We previously demonstrated host-origin of neointimal SMCs in experimental transplant vasculopathy in rats ${ }^{15}$ and more recently in human renal allografts ${ }^{16}$. Direct evidence supporting the contribution of (bone marrow-derived) circulating aSMA expressing cells in the development of human restenosis is lacking. However, human atherosclerotic plaques were shown to contain bone marrow-derived $\alpha$ SMA expressing cell ${ }^{17}$. In line with this, human peripheral blood was shown to contain a population of smooth muscle progenitor cells ${ }^{18,19}$.

Much speculation therefore exists with respect to the precise roles of $\mathrm{T}$ cells in response to arterial injury which is complicated by the fact that researchers use diverse immunodeficiency models and different modes of arterial injury. Nevertheless, based on the available literature we hypothesized that the specific immune response to arterial injury depends on the method, and thereby severity, of arterial injury. Denudation wire-injury is considered a severe method to induce neointima formation. Here the endothelium is removed and the artery stretched due to the size of the guide-wire. The combination of denudation and arterial stretch results in robust neointima formation. The other model used in this study was the perivascular cuff method. Here the artery is sheathed with a polyethylene tube around the femoral artery. The tube is larger than the vessel so that the blood flow is not obstructed. The exact mechanism of neointima formation is currently unknown.

In this study we developed a humanized mouse model which would allow the investigation of: 1) the involvement of human peripheral blood mononuclear cells (PBMCs) in the immune responses and subsequent neointima formation elicited by vascular injury, and 2) the contribution of human PBMC-derived $\alpha$ SMA expressing cells to neointima formation in 2 widely-used models for neointima formation in mice.

\section{Results}

Neointima formation after single vs. dual injury in BL/6 mice. Neointima formation may vary significantly depending on the type of vascular injur ${ }^{20,21}$, including the contribution of circulation-derived $\alpha$ SMA expressing cells to neointima formation ${ }^{22}$. Since we wanted to study the differential effects of arterial injury (i.e., carotid wire versus femoral cuff injury) in NSG mice, we first had to validate the dual injury model and compare data with single injury. Both the carotid wire and femoral cuff injury model are widely used to study neointima formation, but to our knowledge these injuries have not been performed simultaneously in the same mouse. We carried out either single or dual injury in BL/6 mice which resulted in neointimal lesion formation 4 weeks after injury when compared to sham-operated mice (Fig. 1A-F). After single injury, the femoral cuff model appeared to induce slightly more neointima formation (expressed as \% intimal expansion) when compared to carotid wire injury (23\% vs. $28 \%$, respectively, Fig. $1 G)$. No significant differences in intimal expansion in femoral and carotid arteries were observed when comparing single injured and dual injured BL/6 mice (Fig. 1G). Similar to single injury, dual injury resulted in the development of an $\alpha \mathrm{SMA}^{+} \mathrm{SMC}$-rich neointima (Fig. S2B,D). Thinning of the tunica media was observed in the femoral artery 4 weeks after cuff injury (Fig. S2D,E) yet the media of the carotid artery did not significantly differ after wire-injury (Fig. S2B,E). Masson's trichrome staining identified collagen content in the media of the carotid artery after wire-injury (Fig. S2F). Yet, collagen deposition was not found in the neointima of either the carotid or femoral artery after injury (Fig. S2F,G). These data indicate that induction of dual injury does not influence the severity of neointima formation when compared to single injury.

Neointima formation after dual injury in non-reconstituted NSG mice. Next, we investigated the role of immunodeficiency on neointima formation as this has not been studied in NSG mice. Dual arterial injury was carried out in NSG mice and the damaged arteries analyzed 4 weeks later. Unlike our findings in BL/6 mice, no neointima formation was observed in the wire-injured carotid arteries of NSG mice $(\mathrm{P}<0.05 v s$. BL/6 mice, Fig. $1 \mathrm{H})$. In contrast, the femoral artery cuff which was placed in the same mouse did induce neointima formation ( $\mathrm{P}=0.01$ vs. NSG carotid artery) to an extent similar to what was found in immunocompetent $\mathrm{BL} / 6$ mice (Fig. $1 \mathrm{H})$. We therefore investigated the early response to dual vascular injury in NSG mice by examining the arteries of NSG mice 2 days after dual injury. Verhoeff-van Gieson staining (Fig. S3A), Masson's trichrome staining (Fig. S4A-F) and immunofluorescent staining for aSMA (Fig. S3B,C) confirmed thinning of the femoral artery media as a result of cuff placement. In addition, the total number of cells was also reduced after cuff placement (Fig. S3D). In contrast, the media area of the carotid artery slightly increased (Fig. S3C) yet the total number of cells in the media of both the carotid artery was severely reduced 2 days after injury (Fig. S3D). In the femoral artery, a reduction in the number of cells present in the adventitia was also observed but the number of cells remained unchanged in the adventitia of the injured carotid artery (Fig. S3D). The reduction in cell numbers is likely a result of apoptosis early after injury. Staining for vWF confirmed endothelial staining in sham-operated arteries (Fig. S3e.1,e.3). In contrast, only a few $\mathrm{vWF}^{+}$endothelial cells were present in injured carotid and femoral arteries confirming endothelial denudation 2 days after injury (Fig. S3e.2,e.4). At 2 days after injury we found thrombus formation in the carotid arteries of all mice $(n=4)$ (Fig. S3a.2,a.3) but not in the femoral artery arteries (Fig. S3a.5,a.6) which may be due to the differences between the vascular injury methods. In addition, it may also reflect the heterogeneity of the vasculature. Despite the susceptibility for thrombus formation in carotid arteries early after injury, this clearly did not result in neointima formation 4 weeks after injury (Fig. 1H).

Generation of humanized NSG mice: engraftment of human PBMCs. To generate humanized NSG mice, we transferred $5 \times 10^{6}$ human PBMCs isolated from the peripheral blood of 5 healthy male donors to NSG mice. PBMCs from each donor were used to reconstitute NSG mice that received arterial injury $(n=2)$ as well as sham mice $(n=2)$. NSG mice engrafted with human PBMCs are known to eventually develop symptoms of graft-versus-host disease (GvHD) depending on the number of PBMCs transferred. However, minimal GvHD occurs at one month when transferring $5 \times 10^{6} \mathrm{PBMCs}^{23}$. PBMCs were administered immediately after the dual arterial injury procedure was performed and mice sacrificed 28 days later. Reconstituted NSG mice showed 


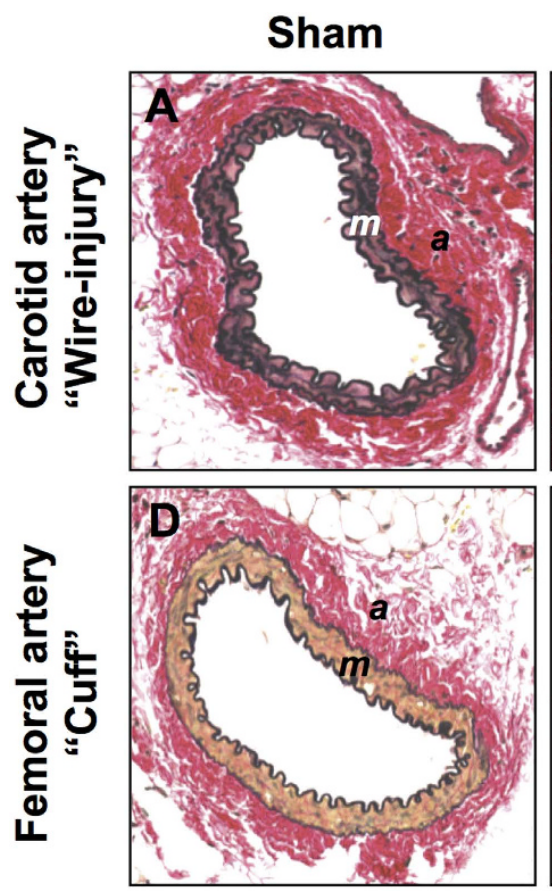

G
Single injury
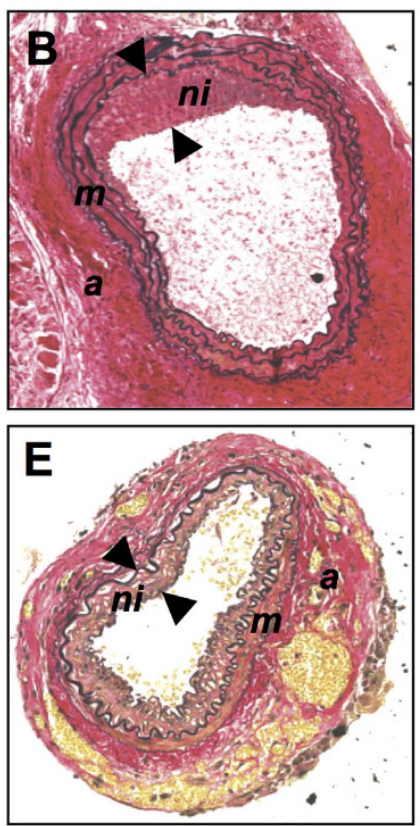

Dual injury
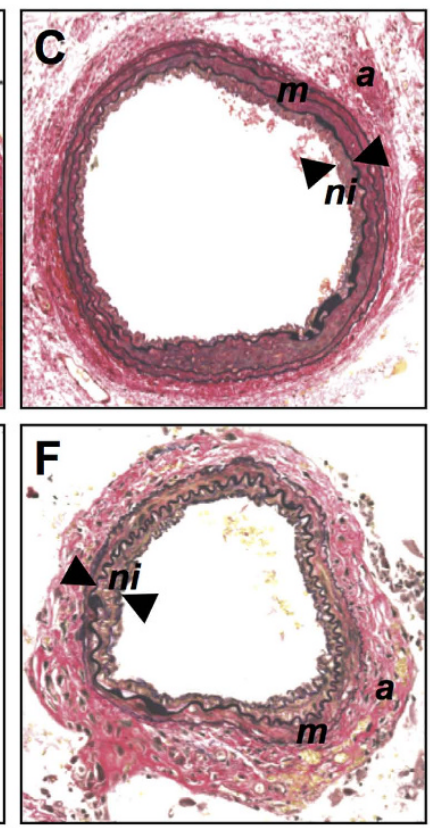

H
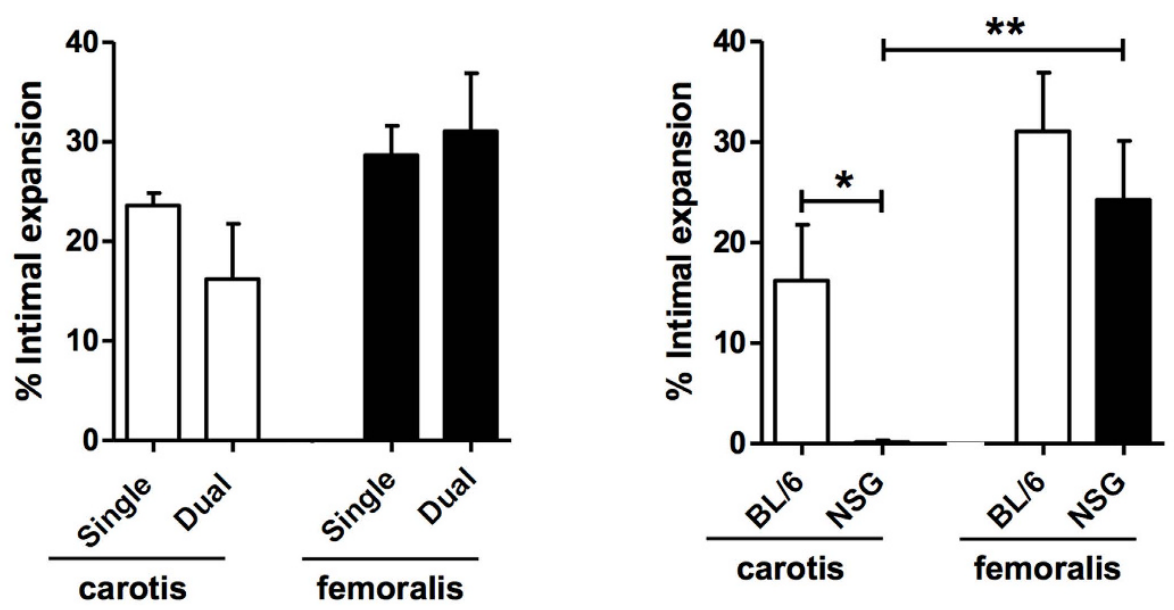

Figure 1. Neointima formation 4 weeks after dual vascular injury in BL/6 and NSG mice. Representative sections of carotid and femoral arteries from dual injured and sham-operated BL/6 mice stained with Verhoeffvan Gieson (A-F). Neointima is indicated as tissue in between arrowheads. (A) Sham-operated control carotid artery, (B) Carotid artery after single wire-denudation injury, (C) Carotid artery after wire denudation in a dual injured mouse, (D) Sham-operated control femoral artery, (E) Single collar-injured femoral artery, and

(F) Collar-injured femoral artery in a dual injured mouse. Abbreviations: $a$ : adventitia; $m$ : media; $n i$ : neointima. (G) Intimal expansion in arteries of single $(n=5)$ and dual $(n=5)$ injured BL/6 mice. No significant difference in intimal expansion was detected when comparing single versus dual injured mice. (H) Intimal expansion measured in the arteries of dual injured BL/6 $(n=5)$ and NSG $(n=9)$ mice. No significant difference was found in the amount of neointima observed between the femoral arteries of BL/ 6 and NSG mice, whereas intimal expansion in the carotid artery of NSG mice was significantly reduced when compared with BL/6 mice $\left({ }^{\star} \mathrm{P}<0.05\right)$. In NSG mice, femoral arteries displayed significant intimal expansion compared with carotid arteries $\left({ }^{\star \star} \mathrm{P}=0.01\right)$. Data are expressed as mean \pm SEM. Original magnification $(\mathbf{A}-\mathbf{F}): 200 \mathrm{x}$.

marked splenomegaly (Fig. 2A). Immunofluorescent labeling for h(uman) CD45 revealed massive splenic repopulation with hCD $45^{+}$cells in engrafted NSG mice, whereas no hCD $45^{+}$were detected in non-reconstituted mice 4 weeks after administration. These data were supported by flow cytometric analyses performed on peripheral blood and spleen 4 weeks after reconstitution (Fig. 2B,C). As expected, no hCD $45^{+}$cells were detected in NSG mice that had not received human PBMCs. Higher levels of engraftment were observed in the spleen (44\%) as compared to that achieved in the blood $(21 \%)$ at 4 weeks $(\mathrm{P}<0.01$, Fig. $2 \mathrm{~B}-\mathrm{D})$. Vascular injury did not alter the amount of engrafted hCD $45^{+}$cells, as similar levels were found in both sham-operated and injured mice 
A

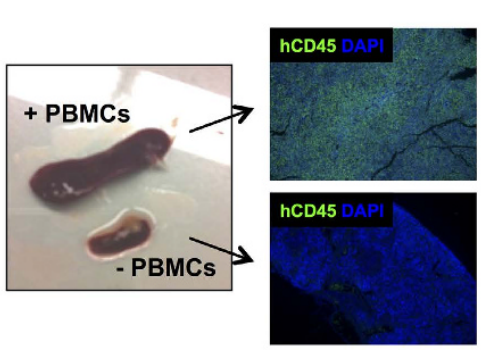

B

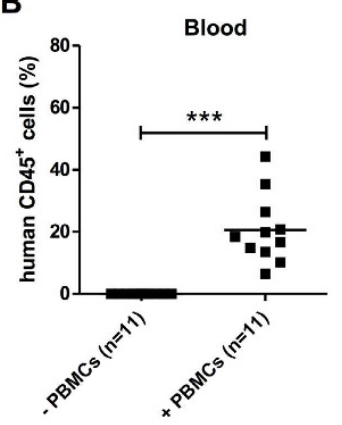

C

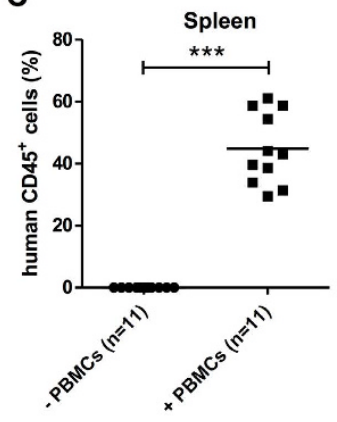

D

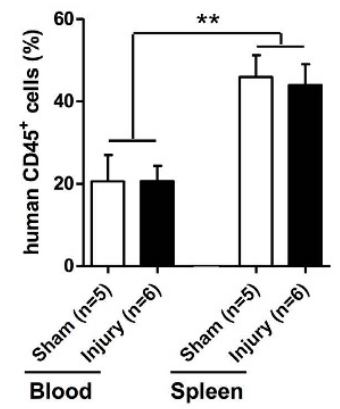

E
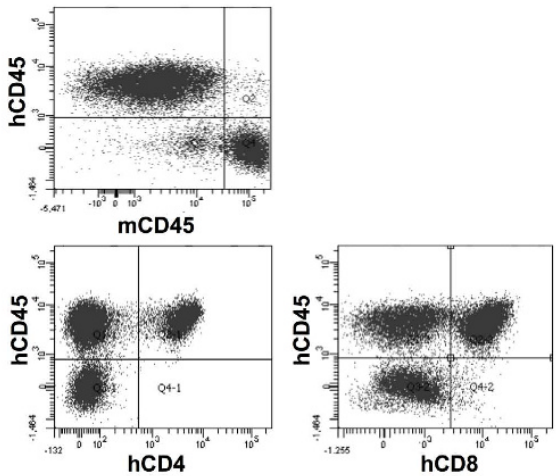

$\mathbf{F}$
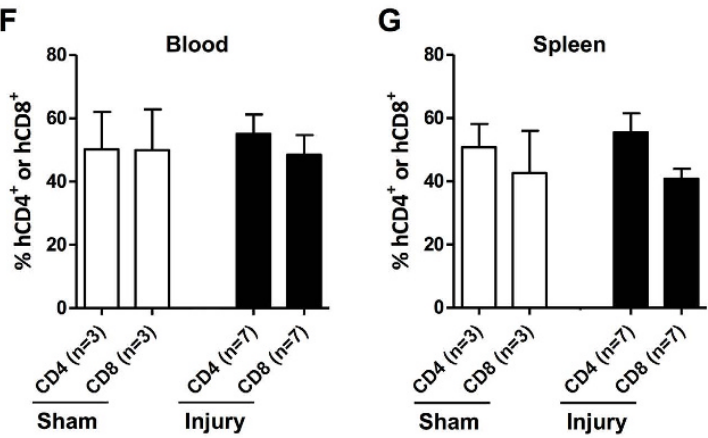

Figure 2. Engraftment of human PBMCs into NSG mice 4 weeks after reconstitution. NSG mice were reconstituted with $5 \times 10^{6}$ human PBMCs by retro-orbital injection. Engrafted spleens showed marked splenomegaly and were repopulated with $\mathrm{hCD} 45^{+}$cells as revealed by immunofluorescent labeling (A). Engraftment of peripheral blood (B) and spleen (C) was evaluated by flowcytometry 4 weeks after reconstitution. Values of individual mice and median engraftment levels are shown $\left({ }^{\star * \star} \mathrm{P}<0.001\right)$. No differences in the engraftment of human $\mathrm{CD} 45^{+}$cells was observed in NSG mice which had first received arterial injury compared to sham-operated mice. Engraftment in spleen was significantly higher than in peripheral blood $\left.{ }^{* *} \mathrm{P}<0.01\right)(\mathrm{D})$. Representative dot plots of human PBMC-engrafted spleens are shown in panel $(\mathbf{E})$. Human $\mathrm{CD}^{+}$and $\mathrm{CD}^{+}$cells were gated on live $\mathrm{hCD} 45^{+}$cells $(\mathrm{E})$ and percentages given represent values of $\mathrm{hCD}^{+}$and $\mathrm{hCD}^{+}$cells within the $\mathrm{hCD} 45^{+}$population in peripheral blood $(\mathbf{F})$ or spleen $(\mathbf{G})$. The $\mathrm{n}$-value reflects the number of mice included. Data are expressed as mean $\pm \mathrm{SEM}$.

(Fig. 2D). Almost all of the engrafted human cells in the blood and spleen were CD3 ${ }^{+}(\sim 99 \%)$ (data not shown). Within the population of hCD $45^{+}$cells the percentages of $\mathrm{hCD} 4^{+}$and $\mathrm{hCD} 8^{+}$cells were determined as depicted in Fig. 2E. The percentages of $\mathrm{hCD}^{+}$and $\mathrm{hCD}^{+}$in the blood and spleen were not significantly different in reconstituted NSG mice which had not received arterial injury compared to mice which had first received arterial injury (Fig. 2F,G). To determine GvHD in the PBMC-transferred NSG mice, we monitored for weight loss and performed haematological analyses at sacrifice. Although weight loss occurred shortly after arterial injury as a consequence of the surgical procedure, all mice had returned to their starting weight within a week and no differences were observed between PBMC-reconstituted and non-reconstituted mice (data not shown). A significantly higher number of white blood cells were detected in PBMC-reconstituted NSG mice (Fig. S5) which corresponds with the engraftment of human $\mathrm{CD} 45^{+}$cells (Fig. 2B). In NSG mice, red blood cell count, haemoglobin, haematocrit, and platelet counts were within the normal range and no differences were observed between reconstituted and non-reconstituted mice (Fig. S5B-E). These results confirm that the reconstituted NSG mice were not suffering from the detrimental effects of GvHD.

Plasma levels of human and mouse cytokines in humanized NSG mice. In order to analyze whether dual vascular injury modulates systemic human PBMC cytokine production in humanized mice which may influence neointima formation, plasma samples were analyzed for cytokine levels using an ultra-sensitive 10-plex multiplex assay capable of detecting very low levels of human inflammatory cytokines. Plasma from both BL/6 mice and non-reconstituted (-PBMCs) NSG mice were used as controls. These control mice had no detectable levels of the human inflammatory cytokines analyzed (Fig. 3A-H). However, low levels of the human cytokines IL-1 $\beta$ (A), GM-CSF (B), IFN $\gamma(\mathrm{C})$, IL-10 (D), IL-2 (G), and IL-5 (H) were measured in the plasma of mice reconstituted with human PBMCs (Fig. 3, white bars). Significantly elevated levels of IL-1 $\beta(P<0.05, A)$, GM-CSF $(\mathrm{P}<0.01, \mathrm{~B}, \operatorname{IFN} \gamma(\mathrm{P}<0.01, \mathrm{C})$, and IL-10 $(\mathrm{P}<0.05, \mathrm{D})$ were observed in PBMC-engrafted mice (compared with non-reconstituted mice) 4 weeks after receiving dual arterial injury (Fig. 3, black bars). Although not significant, increased plasma levels of human IL-2 (G), IL-5 (H), IL-6 (E) and TNF $\alpha$ (F) were also observed in reconstituted mice which had received dual arterial injury compared to uninjured reconstituted mice (Fig. 3). Inflammatory cytokines of mouse origin (IL-1 1 , IL-5 and IL-6) were not detected in the plasma of (non-) reconstituted NSG mice with or without arterial injury (data not shown). 
A

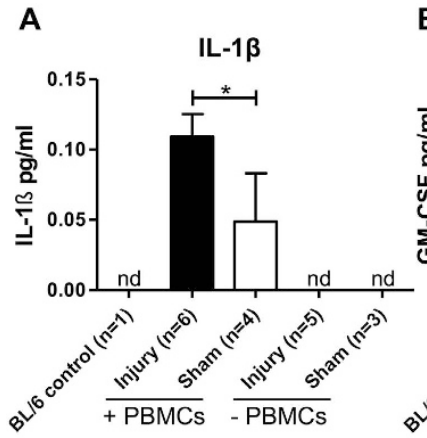

B

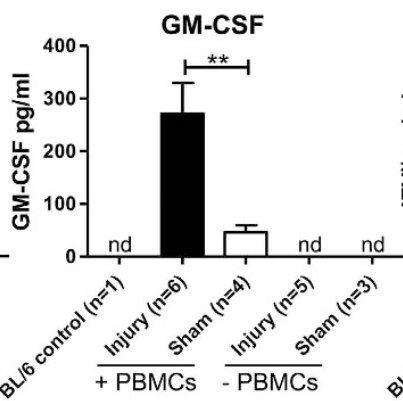

C

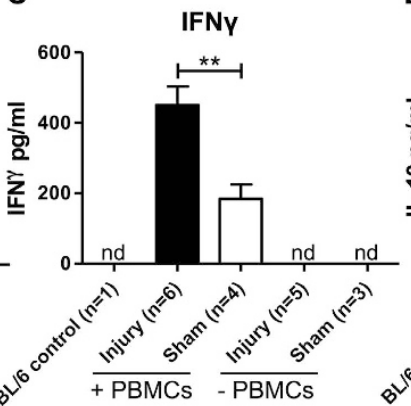

D

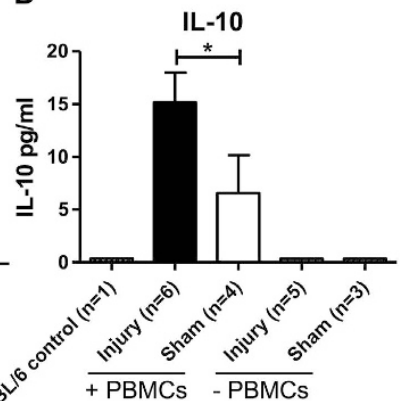

E

F

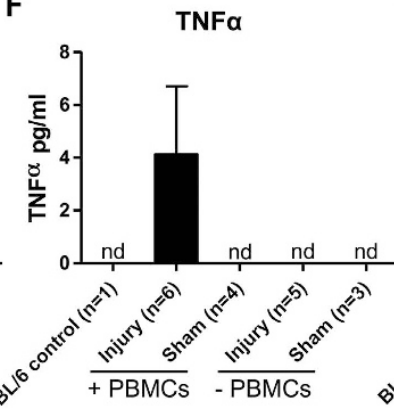

IL-6

G IL-2

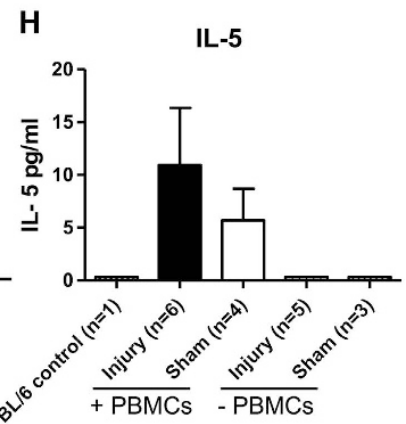

Figure 3. Plasma human cytokine levels in NSG mice reconstituted with $(+)$ and without $(-) 5 \times 10^{6}$ human PBMCs, and with and without (sham) arterial injury. Plasma concentrations of human IL-1 $\beta$ (A), GM-CSF (B), IFN $\gamma(\mathbf{C})$, IL-10 (D), IL-6 (E), TNF $\alpha$ (F), IL-2 (G) and IL-5 (H) were analyzed using an ultrasensitive cytokine bead array 4 weeks after PBMC reconstitution and arterial injury. Significant differences between injured and sham-operated reconstituted mice were found for IL-1 $\beta$, GM-CSF, IFN $\gamma$ and IL-10. The $\mathrm{n}$-value reflects the number of mice included. Data are expressed as mean $\pm \mathrm{SEM} .{ }^{\star} \mathrm{P}<0.05,{ }^{\star \star} \mathrm{P}<0.01$. (nd $=$ not detectable; $\mathrm{ns}=$ not significant).

Neointima formation in dual injured PBMC-engrafted and non-reconstituted NSG mice. We next examined the effect of human PBMC reconstitution in NSG mice on neointima formation after dual arterial injury. Significant neointima formation was found in the carotid artery of reconstituted NSG mice four weeks after arterial injury yet no neointimal lesions were observed in carotid arteries of NSG mice without human cell reconstitution ( $\mathrm{P}<0.01$ vs. non-reconstituted NSG, Fig. 4A,C,E,G). In contrast, neointima formation was significantly reduced in the femoral arteries of reconstituted NSG mice when compared with non-reconstituted mice $(\mathrm{P}<0.05$, Fig. 3B,D,F,G). Similar results were obtained when expressing data as Intima/Media ratio (Fig. $4 \mathrm{H})$.

Smooth muscle cell phenotypic modulation after arterial injury. In order to determine the SMC phenotype of neointimal cells in injured carotid and femoral arteries we performed immunofluorescent double labeling for $\alpha \mathrm{SMA} / \mathrm{calponin}$ and $\alpha \mathrm{SMA} / \mathrm{SM} 22 \alpha$. Non-injured carotid and femoral arteries were used as positive controls since differentiated medial SMCs within these arteries are known to express $\alpha$ SMA, SM22 $\alpha$ and calponin (Fig. 5A-D, left panels). Medial SMCs in injured arteries also expressed all three SMC markers (Fig. 5A-D, right panels). In contrast, neointimal lesions in both the carotid and femoral arteries were characterized by low $\alpha$ SMA expression accompanied with reduced expression of SM22 $\alpha$ and calponin supporting a de-differentiated proliferative SMC phenotype. Production of excessive connective tissue as a result of SMC phenotypic modulation from the contractile-type to the de-differentiated synthetic-type is proposed to play a major role in the response to vascular injury. Trichrome staining (Martius Scarlet Blue [MSB] revealed minimal collagen content in the media of sham-operated carotid (Fig. S4A,G) and femoral arteries (Fig. S4D,J). In contrast, MSB staining revealed extensive collagen deposition in the neointima of carotid arteries (Fig. S4I) and in the media of femoral arteries (Fig. $\mathrm{S} 4 \mathrm{~L}$ ) of reconstituted mice 4 weeks after injury. In immunodeficient mice, collagen deposition in the neointima was minimal after femoral artery cuff injury (Fig. S4K) and was absent in the media of the carotid artery after wire-injury (Fig. S4H). Hence, SMC phenotypic modulation was independent of the immune status of the mice. However, in contrast to immunocompromised (no PBMCs) mice, collagen deposition found in the neointima and media of injured arteries appeared to be more extensive in the arteries of reconstituted mice.

Human leukocyte infiltration in dual injured PBMC-engrafted NSG mice. In order to determine if the differences in neointima formation in injured femoral and carotid arteries in PBMC-reconstituted and non-reconstituted NSG mice were associated with human leukocyte infiltration, double immunofluorescent labeling was performed on arterial sections using human-specific antibodies against pan-leukocytes (hCD45), T cell subsets (hCD4 and hCD8) and macrophages (hCD68) combined with $\alpha$ SMA. The species specificity of the human-specific antibodies was confirmed by staining spleen sections of PBMC-reconstituted and non-reconstituted NSG mice as well as human appendix (positive control) with the appropriate antibodies 

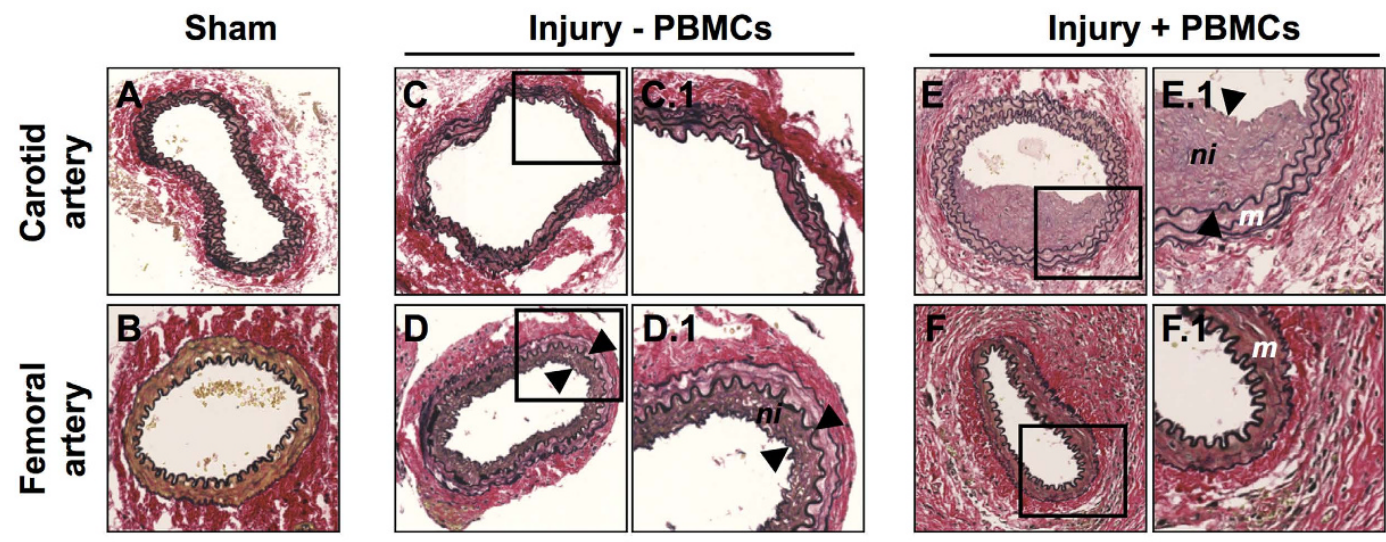

G

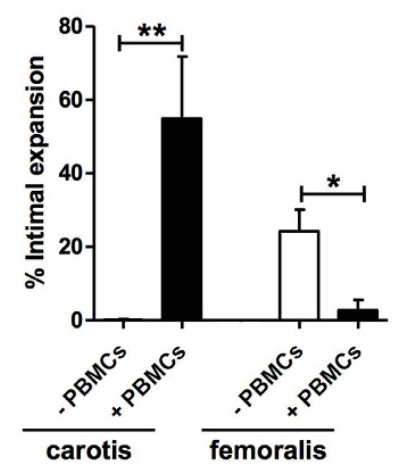

H

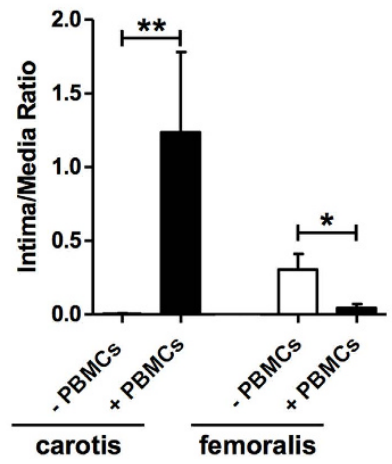

Figure 4. Neointima formation 4 weeks after dual vascular injury in NSG mice reconstituted with or without human PBMCs. Representative sections of carotid and femoral arteries from dual injured and shamoperated mice stained with Verhoeff-van Gieson. (A) Sham- operated control carotid artery, (B) Sham-operated control femoral artery, (C) Carotid artery after wire injury in a non-reconstituted NSG mouse, (D) Femoral artery after cuff injury in a non-reconstituted NSG mouse, (E) Carotid artery after wire injury in a human PBMC-reconstituted NSG mouse, (F) Femoral artery after cuff injury in a human PBMC-reconstituted NSG mouse. Squares indicate areas shown at higher-power magnification of the respective cross-sections (C.1, D.1, E.1, F.1). Neointima is indicated as tissue in between arrowheads. Neointima formation in the carotid and femoral arteries of dual injured NSG mice reconstituted with $(n=6)$ and without $(n=9)$ human PBMCs was expressed as \% intimal expansion $(\mathbf{G})$ and intima/media ratio $(\mathbf{H}) .{ }^{\star} \mathrm{P}<0.05,{ }^{* *} \mathrm{P}<0.01$. Data are expressed as mean \pm SEM. Abbreviations: $m$ : media; ni: neointima; PBMCs: human peripheral blood mononuclear cells. Original magnification (A-F): 200x.

using immunohistochemistry (Fig. S6) and immunofluorescence (Fig. S7). Staining of injured carotid artery sections revealed the presence of human PBMC-derived hCD $45^{+}$leukocytes in large numbers in the adventitia but also within the neointima 4 weeks after injury (Fig. 6A). The majority of these hCD $45^{+}$cells appeared to be hCD8 ${ }^{+}$(Fig. 6C), whereas only few $\mathrm{hCD}^{+}$cells were observed within the neontima (Fig. 6B). Moreover, no human-derived $\mathrm{CD}^{+} 8^{+}$macrophages were found within the neointima, media or adventitia of the injured carotid artery (Fig. 6D). In PBMC-reconstituted mice virtually no neointimal lesions were present within the femoral arteries of the same dual injured mice. In the adventitia of these arteries hCD $45^{+}$were present (Fig. $6 \mathrm{E}$ ) which were found to be primarily hCD8 ${ }^{+}$cells (Fig. 6G). No hCD $4^{+}$or hCD68 ${ }^{+}$cells were found (Fig. 6F,H).

No human-PBMC derived SMCs and ECs in dual injured PBMC-engrafted NSG mice. Neointimal SMCs expressing $\alpha$ SMA may be in part derived from circulating presumed myeloid, progenitor cells. To analyze whether in our humanized mouse model PBMC-derived $\alpha$ SMA expressing neointimal cells were present, double labeling was performed for hCD45 (as well as hCD4, hCD8 and hCD68) and $\alpha$ SMA. Although hCD45 ${ }^{+}$cells were present in the $\alpha \mathrm{SMA}^{+}$neointima, clearly no $\mathrm{CD}_{4} 5^{+} \alpha \mathrm{SMA}^{+}$double positive cells were detected (Fig. 6A). The same was true for the other markers included (hCD4, hCD8 and hCD68, Fig. 6B-D). In addition, high resolution confocal microscopy revealed no co-expression of hCD45 or hCD8 with SMA ${ }^{+}$neointimal SMCs (Fig. 6I,J). These data suggest that the $\alpha \mathrm{SMA}^{+}$SMCs in carotid neointimal lesions in humanized NSG mice are not derived from human PBMCs.

In Fig. S3 we showed endothelial denudation in both carotid and femoral arteries early after injury. This observation raised the question whether re-endothelialization is mediated (in part) by endothelial progenitor cells which are known to reside within the PBMC fraction. To address this question, species-specific immunofluorescence was performed using antibodies directed against hCD31 and mCD31. The specificity of the hCD31 
A

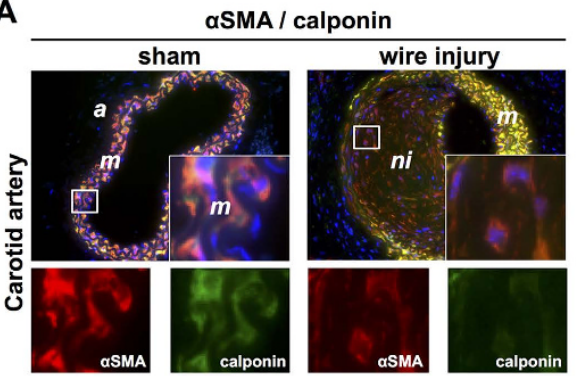

C

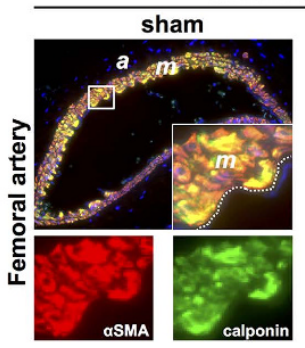

aSMA / calponin

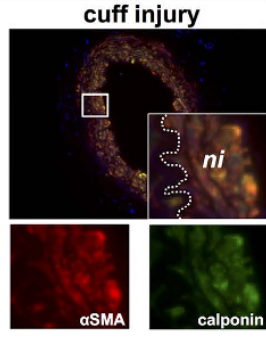

B

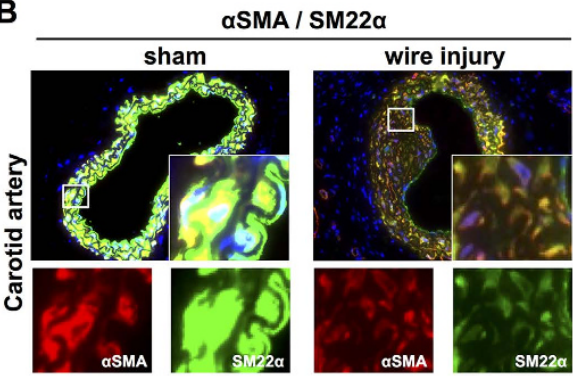

D

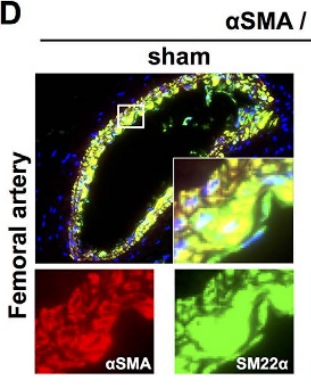

aSMA / SM22a

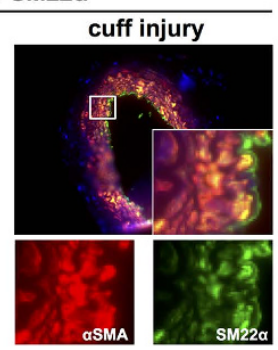

E

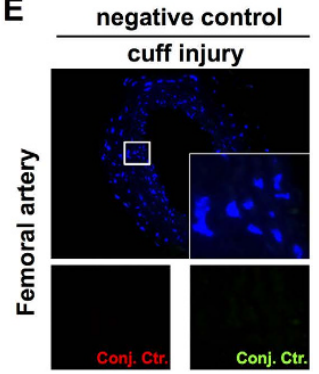

Figure 5. Neointimal cells in the injured carotid (PBMC-engrafted NSG mice) and femoral (non-PBMCengrafted NSG mice) artery express $\alpha$ SMA, calponin and SM22 $\alpha$. Sham-operated (non-injured) and wire-injured carotid arteries stained for $\alpha$ SMA/calponin (A) and $\alpha$ SMA/SM22 $\alpha$ (B). Sham-operated and cuff-injured femoral arteries stained for $\alpha$ SMA/calponin (C) and $\alpha \mathrm{SMA} / \mathrm{SM} 22 \alpha(\mathbf{D})$. As negative control, cuff-injured femoral arteries were incubated according the protocol but with omitting primary antibody incubation (E). Insets and single stainings show high power magnifications of the boxed regions. Acquired photomicrographs were converted into pseudocolored images and merged using ImageJ $1.47 \mathrm{v}$ (http://imagej. nih.gov/ij). Abbreviations: $a$ : adeventitia; $m$ : media; ni: neointima.

antibody was confirmed on human venous tissue (Fig. S7B). In sham-operated carotid and femoral arteries, both in non-reconstituted (Fig. 7A,I) and PBMC-reconstituted (Fig. 7C,K) NSG mice the endothelium was of mouse origin. Also 4 weeks after injury, both in non-reconstituted (Fig. 7B,J) and PBMC-reconstituted (Fig. 7D,L) mice the endothelium was of mouse origin. Human PBMC-derived ECs were never detected in carotid (Fig. 7E-H) and femoral (Fig. 7M-P) arteries. Similar to neointimal SMCs, these data indicate that ECs in injured arteries in humanized NSG mice are not derived from human PBMCs.

Neointima formation in the femoral artery after wire-injury. To determine whether the type of artery or the method of inducing injury (wire vs cuff) is important we also performed wire-injury in the femoral artery of NSG mice with or without PBMC reconstitution. Four weeks after surgery and reconstitution the level of chimerism in the peripheral blood was about $22 \%$ (Fig. 8A) consistent with our other studies (Fig. 2B). In contrast to the carotid artery, femoral artery wire injury resulted in neointima formation already without reconstitution. Furthermore, reconstitution with hPBMCs did not attenuate neointima formation in wire-injured femoral arteries (Fig. 8B,C). Hence, neointima formation in the femoral artery of immunocompromised mice is not dependent on the type of vascular injury since both the cuff and wire injury resulted in around $20 \%$ neointimal expansion.

\section{Discussion}

The mechanisms underlying restenosis development following PCI remain unclear. As a consequence additional experimental models are needed to investigate the interaction between the (human) immune system and the vascular wall in neointima formation. In the current study we developed a humanized mouse model based on the immunodeficient NSG mouse strain. NSG mice lack functional T cells, B cells, and NK cells. Moreover NSG mice have functional defects in macrophages, and dendritic cells and have no hemolytic complement ${ }^{24,25}$. Reconstitution of NSG mice enabled us to investigate the involvement of human PBMCs in the immune responses and subsequent neointima formation elicited by vascular injury, and, secondly, to determine the contribution of human PBMC-derived $\alpha$ SMA expressing cells to neointima formation. The severity of neointima formation and the contribution of circulating cells to neointimal lesions varies depending on the type of vascular injury $\mathrm{y}^{20-22,26}$. We therefore applied two commonly used experimental methods of vascular injury, i.e. carotid artery wire injury and femoral artery cuff injury, together in the same mouse to study the effect of human PBMC reconstitution on neointima formation. Wire injury results in endothelial denudation and is known to disrupt the internal elastic lamina and the media whereas placement of a polyethylene cuff loosely over the femoral artery stimulates slow sloughing of the endothelium within 1-2 days after placement ${ }^{27}$. We hypothesized that differences in the severity of injury and the exposed denuded vasculature would influence endothelial regeneration, homing of inflammatory and progenitor cells, and the proliferation of medial SMCs. 


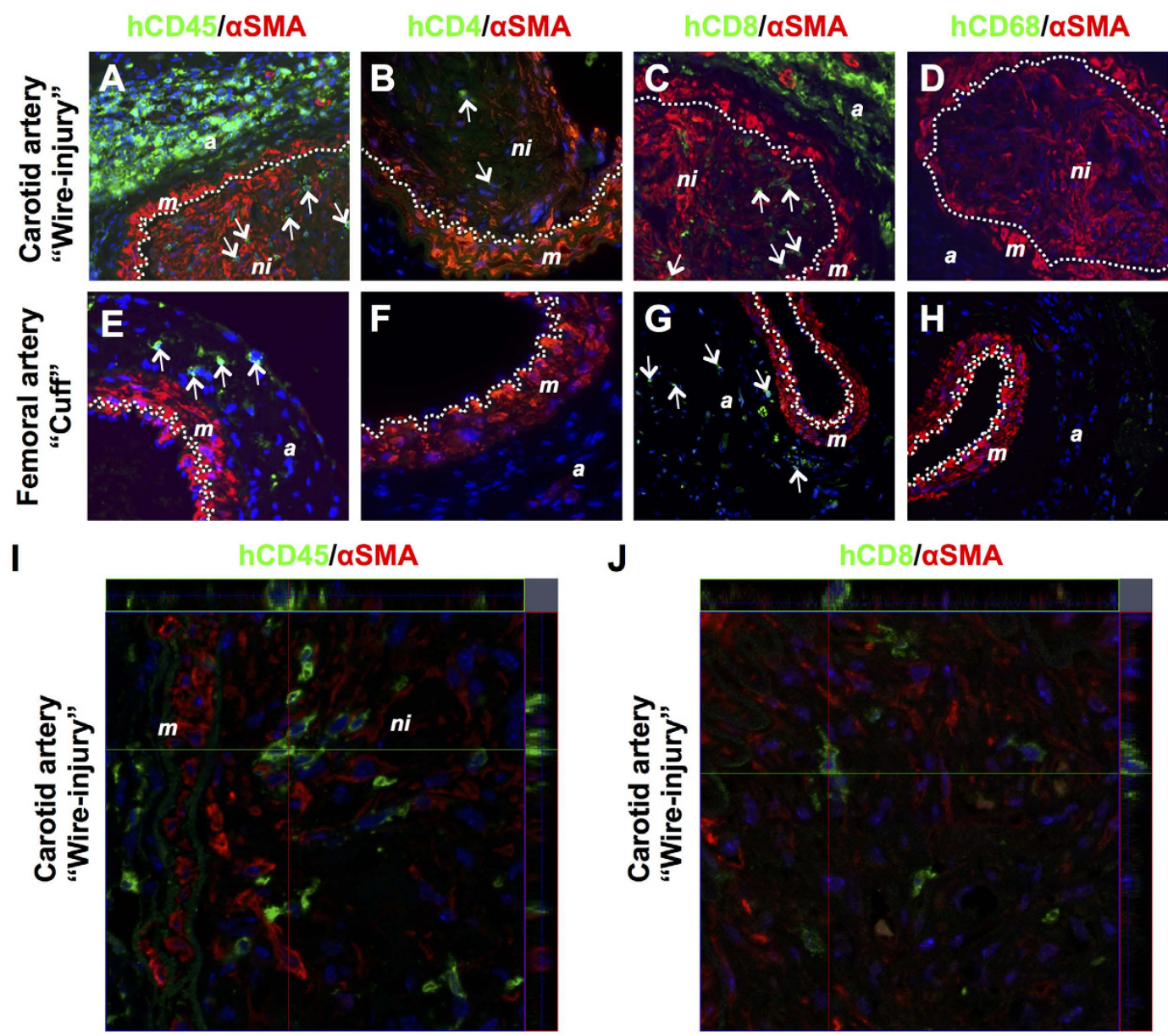

Figure 6. Presence of human PBMC-derived cells within injured carotid (A-D) and femoral (E-H) arteries in NSG mice 4 weeks after reconstitution with $5 \times 10^{6}$ human PBMCs. Sections were immunofluorescently stained with $\alpha$ SMA (in red) and specific antibodies (in green) recognizing human CD45 (A,E), human CD4 (B,F),

human CD8 $(\mathbf{C}, \mathbf{G})$ and human CD68 (D,H). Representative images of arterial staining from 3 different mice per group. Specificity controls for the antibodies used are shown in Figs S6 and S7A. Nuclei were stained with DAPI (in blue). Dotted line indicates the internal elastic lamina. Arrows indicate positively stained human leukocytes.

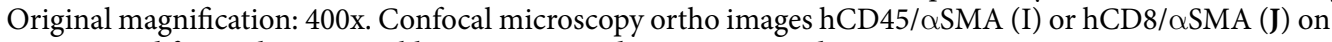
wire-injured femoral arteries. Abbreviations: $a$ : adventitia; $m$ : media; $n i$ : neointima.

Dual injury in immunocompetent BL/6 mice resulted in neointima formation in both the carotid and femoral arteries. However, in non-reconstituted NSG mice significant neointima formation was observed only in the femoral artery 4 weeks after cuff placement. This observation is in line with previous studies in immunodeficient Rag $^{-1-}$ mice also showing intimal hyperplasia after femoral artery injury ${ }^{7,8}$. In contrast, we surprisingly found no neointima formation in the carotid artery of the same non-reconstituted NSG mice. We had expected substantial neointima formation after wire-induced injury in the carotid artery since this model is thought to be more severe when compared to the perivascular cuff model of the femoral artery ${ }^{28}$. From these results we concluded that immunodeficiency protects against neointima formation which is dependent on the type of injury. To our knowledge, this is the first study that describes the differential (both protective and stimulatory) effects of immunodeficiency on simultaneous carotid wire-injury and femoral artery cuff-injury induced neointima formation in mice.

We next examined the effect of reconstitution on neointima formation after dual arterial injury in NSG mice. High levels of human lymphocyte engraftment with primarily $\mathrm{CD}^{+} \mathrm{T}$ cells were observed in peripheral blood and spleen 4 weeks after reconstitution. In contrast to what we observed in NSG mice without reconstitution, we found that the femoral artery in humanized mice developed no neointima 4 weeks after arterial injury whereas the carotid artery developed severe neointimal lesions. These results indicate that human cell reconstitution, which was predominantly composed of T cells, protects against neointima formation in the femoral artery after cuff injury but not after wire-induced injury, and promotes neointima formation in the carotid artery after wire-induced injury.

Since femoral and carotid arteries are diametrically different (i.e. femoral arteries are approximately 3 times smaller in diameter), the severity of vascular injury induced by the guide wire is most likely different (i.e. increased damage in femoral artery), and consequently the remodeling response. Carotid arteries have 3 layers of elastin and 2 layers of SMCs, whereas the femoral artery has 2 layers of elastin and only one layer of SMCs. In 
- PBMCs
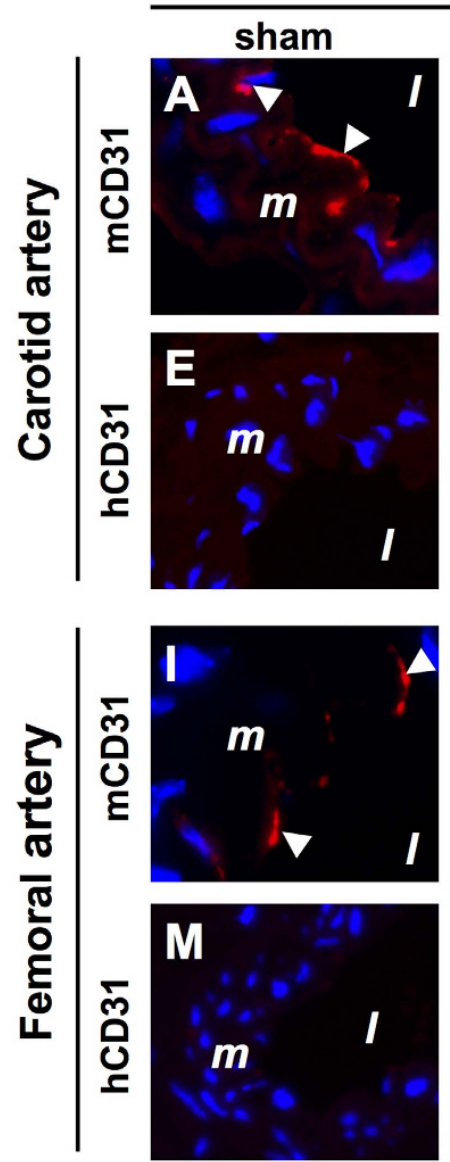

\section{+ injury}
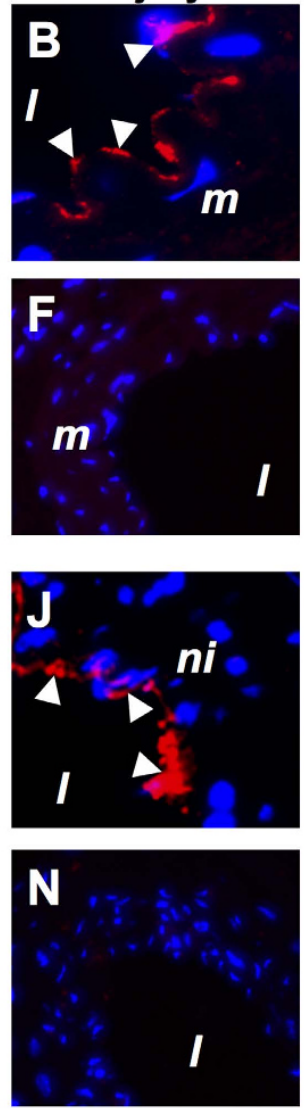

+ PBMCs
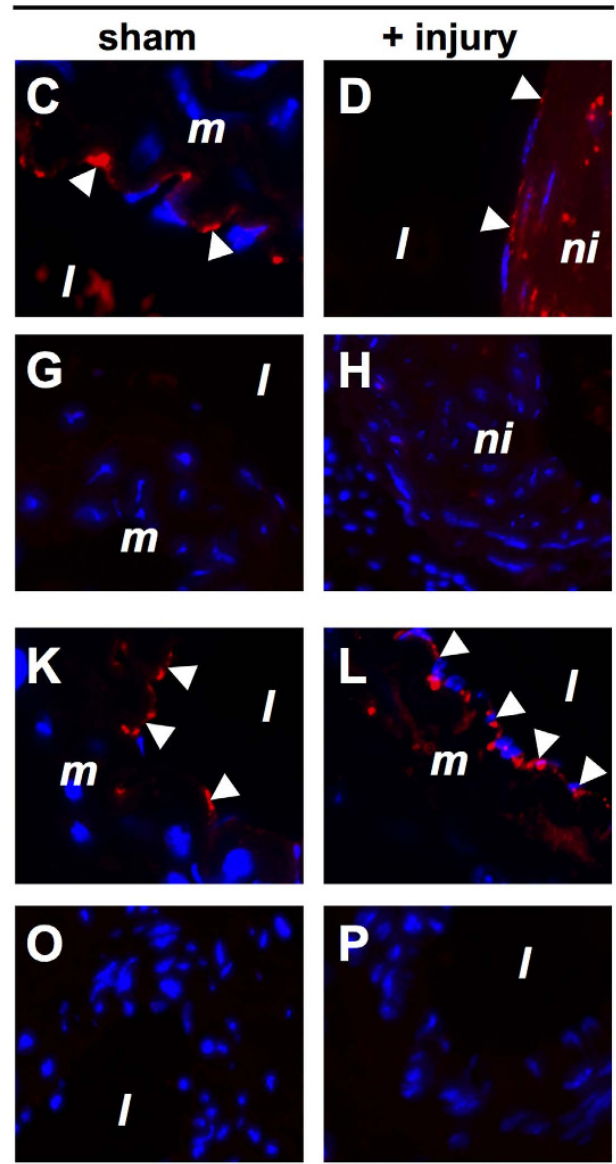

Figure 7. No re-endothelialization of carotid (A-H) and femoral (I-P) arteries with human PBMC-derived CD31+ endothelial cells in NSG mice 4 weeks after reconstitution with $5 \times 10^{6}$ human PBMCs. Sections from sham-operated and injured carotid and femoral arteries obtained from both human PBMC reconstituted (+PBMCs) and non-reconstituted (-PBMCs) NSG mice were included in this analysis. Sections were immunofluorescently stained with anti-human CD31 (hCD31) and anti-mouse CD31 (mCD31) species-specific antibodies. Representative images of arterial staining from 3 different mice per group. Specificity controls for the hCD31 antibody is shown in Fig. S6B. Nuclei were stained with DAPI (blue). Arrowheads indicate positively stained $\mathrm{CD}_{3} 1^{+}$endothelial cells. Abbreviations: l: lumen; $m$ : media; ni: neointima; PBMCs: human peripheral blood mononuclear cells. Original magnification: 400x.

addition to the amount of media (SMCs), vessel elasticity, hemodynamic responses, the basement membrane and other factors such as residual endothelium play important roles in driving the response to arterial injury. Based on our results we could not discriminate whether the type of artery or the method of inducing injury is the most important factor in driving neointima formation in immunodeficient NSG mice. We therefore also performed femoral artery wire injury in NSG mice with or without PBMC reconstitution. Wire-injured femoral arteries developed neointimal lesions to a similar extent as that observed in femoral cuff injured arteries (approximately $20 \%$ intimal expansion) in NSG mice without reconstitution. These results suggest that neointima formation in the femoral artery is not dependent on the type of vascular injury in immunocompromised mice. Moreover, because of the severity of wire-injury (denudation and stretching) in the femoral artery we speculate that occlusion may not be able exceed more than $20 \%$ intimal expansion which is likely attributed to an intrinsic property of the femoral artery itself. Besides, the carotid artery is composed of a larger SMC-rich media compared to the femoral artery which may explain why the carotid artery has more neointima formation compared to the femoral artery after injury. In reconstituted NSG mice, wire-injury of the femoral artery did not attenuate neointima formation (20\% intimal expansion) compared to femoral cuff arteries with only $4 \%$ intimal expansion. These results are in line with substantial neointima formation observed in the carotid artery after wire injury in reconstituted NSG mice. Likely the immediate and complete removal of the endothelial layer in the femoral artery after wire-injury promotes the recruitment of the hPBMCs which were systemically administered to the mice immediately after the surgical procedure. These results suggest that direct recruitment of hPBMCs to the injured arterial wall has detrimental effects (i.e. enhancing or loss of protective effects) which drive neointima formation in the denuded arteries. In contrast, EC removal is slower by the perivascular cuff which likely results in the differential 
A

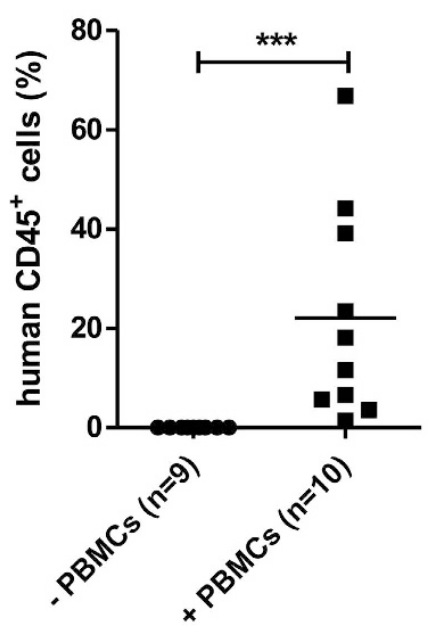

B

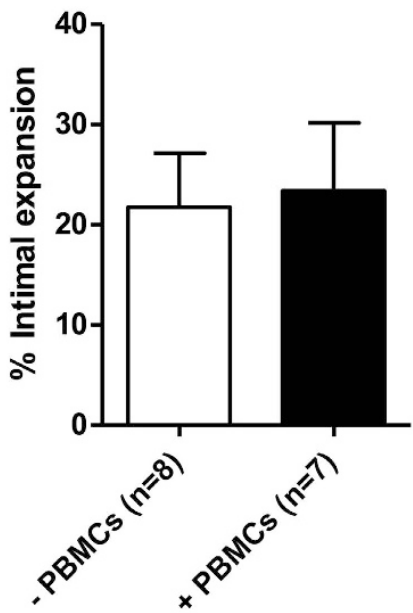

C

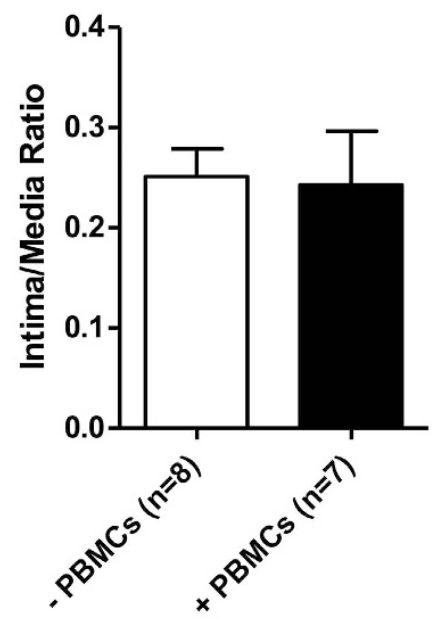

Figure 8. Neointima formation 4 weeks after femoral artery wire injury in NSG mice reconstituted with or without human PBMCs. (A) NSG mice reconstituted with $5 \times 10^{6} \mathrm{hPBMCs}$ and engraftment of peripheral blood was evaluated by flow cytometry 4 weeks after reconstitution. (B) Neointima formation in the wire injured femoral arteries of NSG mice reconstituted with $(n=7)$ and without $(n=8)$ hPBMCs was expressed as $\%$ intimal expansion and $(\mathbf{C})$ intima/media ratio ${ }^{* *} \mathrm{P}<0.001$. Data are expressed as mean $\pm \mathrm{SEM}$.

effects of hPBMCs on neointima formation seen in our study. Together, these results importantly highlight the complexity of neointimal lesion formation and the role of $\mathrm{T}$ cells in this process and suggest that the immune system may differentially respond to arterial injury depending on the method of injury, which may also be influenced by the intrinsic properties of the arteries themselves.

After arterial injury apoptosis may play an important role on limiting vascular SMC proliferation and corresponding neointima formation. However, excessive apoptosis can lead to necrosis and inflammation. In a rat arterial injury model, 2 waves of apoptosis were identified, early from $30 \mathrm{~min}$ until 6 hours after injury initiation, and late apoptosis around 2 weeks after arterial injury ${ }^{29}$. Early apoptosis can lead to inflammation which is thought to contribute to the remodelling process. We found severely reduced numbers of SMCs in the media of the carotid and femoral arteries at 2 days after injury. In addition, adventitial cells were also reduced. This reduction in the amount of cells is likely due to apoptosis.

In our study, no human PBMC-derived neointimal SMCs were detected, indicating that the $\alpha \mathrm{SMA}^{+}$neointimal SMCs were of mouse origin. This is remarkable as it was previously shown that endovascular wire-injury in mice results in neointimal lesions containing significant numbers $(\sim 27 \%)$ of bone marrow-derived $\alpha \mathrm{SMA}^{+}$ cells $^{22}$. A possible explanation might be that engrafted PBMCs in NSG mice are primarily T cells whereas it has been shown that circulating smooth muscle progenitor cells probably reside within the myeloid population ${ }^{18,19}$. Moreover, no human PBMC-derived ECs were detected in the injured femoral and carotid arteries. These data are in line with a report by Hagensen and colleagues in which it was shown that circulating endothelial progenitor cells do not contribute to endothelial regeneration after murine arterial injury ${ }^{30}$.

Immune cells play a crucial role in the formation of neointimal lesions by inducing the production of cytokines and chemokines that may affect EC and SMC survival, proliferation, migration and activation. As expected, we were unable to detect cytokines of mouse origin in any of the experimental NSG groups since these mice have impaired cytokine signaling. In contrast, 4 weeks after reconstitution non-injured NSG mice had basal levels of several human cytokines, yet mice that had received dual arterial injury had significantly higher levels of IL-1 $\beta$, GM-CSF, IFN $\gamma$ and IL-10. These data indicate that vascular injury triggered a functional cytokine release response in humanized mice. The contribution of these cytokines to neointima formation is not fully elucidated but manipulation of cytokines is a potential therapeutic option for the prevention or treatment of restenosis. This is also supported by clinical data obtained with sirolimus-eluting stents which significantly reduced in stent restenosis $^{31}$. Cytokines such as IFN $\gamma$ are thought to increase pro-inflammatory activity following coronary intervention and might therefore be related to the long-term complications after intervention ${ }^{32,33}$. Likewise, GM-CSF has been implicated in atherogenesis ${ }^{34}$ and has been shown to have both pro- and anti-atherogenic properties ${ }^{35}$ whereas GM-CSF deficiency in mice was shown to delay neointima formation after arterial injury ${ }^{36}$. Similarly, IL-10 is an anti-inflammatory cytokine with anti-atherogenic potential which has been shown to inhibit neointima formation after femoral artery injury in hypercholesterolemic mice ${ }^{37-39}$ and rabbits ${ }^{40}$. This IL-10-induced attenuation of neointima formation was attributed to its powerful inhibitory effects on the activation and growth of SMCs. Based on these published data we suspect that the increased cytokine levels, as detected in engrafted NSG mice after injury, may have had a direct effect on SMC behaviour and resulting neointima formation. However, a number of species-specific factors in mice are not cross-reactive with human cells and vice-versa. Therefore, the pro- and anti-inflammatory factors of human origin measured in the plasma of our mice may have 
had no influence on the neointima formation observed in this study. Human GM-CSF and IFN $\gamma$ are known to be only biologically active on human cells $s^{41,42}$ and therefore probably have little influence on neointimal lesion formation after dual arterial injury in mice. However, human IL-10 does have cross-species activity and is known to be active on mouse cells $s^{43,44}$. We therefore presume that the IL-10 measured in our mice would have had an influence on the neointima formation observed in the current study and may be protecting against neointima formation after femoral artery cuff injury as observed in previous studies ${ }^{36}$. However, further studies are required to confirm if this is indeed the case.

Animal models of restenosis provide a tool for investigating the pathophysiological mechanisms as well as translational approaches to vascular interventions. Several murine experimental models exist, carotid and femoral artery wire injury are widely accepted as the most appropriate techniques in studies of post-angioplasty restenosis because it closely resembles the angioplasty procedure that injures both the endothelium and vessel wall driving neointima formation. The perivascular cuff method does not exactly mimic what happens in humans undergoing percutaneous coronary interventions but is widely used in the literature as a method to understand neointima formation. Moreover, it may be adopted as a model system for testing the perivascular delivery of therapeutic compounds in order to inhibit restenosis. Many conclusions are drawn from mouse models of arterial injury which have contributed to our general understanding of the mechanisms promoting neointima formation. However, whether these translate directly to the human situation is unclear. Our findings emphasize the importance of using the most accurate experimental model when investigating mechanisms of neointima formation and preclinical intervention studies.

Most studies to date described the effects of the normal immune system on neointima formation although it is known that immune cell function might be impaired in subjects at increased cardiovascular risk. A strength of the humanized mouse model described here is that this model can be adopted to understand the immune responses of vascular injury in populations which have a high risk of developing restenosis, e.g. diabetes ${ }^{45,46}$. Recent data indicate that immune and progenitor cells from type 2 diabetic subjects are naturally skewed towards pro-inflammatory subsets that likely promote chronic inflammation ${ }^{46}$. Understanding the unique aspects of $\mathrm{T}$ cells from diabetic patients as well as understanding their influence on neointima formation is essential in order to develop pharmaceutical agents to target restenosis.

In conclusion, we have developed a humanized NSG dual vascular injury model which allows us to study the contribution of the $\mathrm{T}$ cell system on neointima formation. We propose that the immune system differentially responds to arterial injury depending on the severity of injury which may also be influenced by vascular heterogeneity.

\section{Methods}

C57BL/6 and NOD.Cg-Prkdc scid/L2rg ${ }^{\text {tm1Wjl }}$ mice. Male 10-week-old C57BL/6 (BL/6) mice were purchased from Harlan (Horst, The Netherlands), housed under standard housing conditions and given free access to

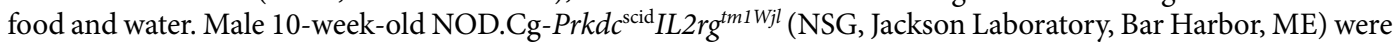
housed in a specific pathogen-free facility in micro-isolator cages and given free access to autoclaved food and water. All experimental procedures were performed according to European Commission guidelines and Dutch laws and were approved by the animal ethics committee of the University of Groningen.

Mouse carotid wire injury and femoral cuff injury models. In this study two different types of vascular injury were induced in the same BL/6 or NSG mouse. Both left common carotid artery (wire-injury) and right femoral artery (femoral cuff injury) of each mouse were injured to induce neointima formation. In contrast to carotid wire-injury, in the femoral cuff model the endothelial cells are not directly manipulated or removed. Mice were anesthetized with $2 \%$ isoflurane $/ \mathrm{O}_{2}$ followed by carotid or femoral artery endothelial denudation and/ or femoral artery cuffing. Briefly, the left carotid artery and its muscular branch or the left femoral artery was exposed, and a $0.014^{\prime}(0.36 \mathrm{~mm})$ diameter angioplasty guide-wire (Cook Medical Europe Ltd., Limerick, Ireland) introduced into the arterial lumen and pulled back three times. Removal of the endothelium was confirmed by in vivo Evan's blue staining in injured arteries compared with uninjured arteries (data not shown). For induction of cuff femoral artery injury, a non-constrictive polyethylene cuff $(0.4 \mathrm{~mm}$ inner diameter, $0.8 \mathrm{~mm}$ outer diameter, length $2 \mathrm{~mm}$, Portex, Kent, UK) was loosely placed around the right femoral artery. Contra-lateral carotid and femoral arteries as well as sham-operated mice were used as controls. Mice were euthanized at two days or four weeks after injury by deep anesthesia $\left(5 \%\right.$ isoflurane $\left./ \mathrm{O}_{2}\right)$ and exsanguination. Spleen and peripheral blood (collected in EDTA vacutainers (BD Biosciences, Franklin Lakes, NJ, USA)) were obtained, plasma was isolated and stored at $-80^{\circ} \mathrm{C}$ until use. After subsequent in situ cardiac perfusion with zinc fixative ${ }^{47}$, arteries were collected for histology. Haematological analyses were performed using the Poch-100i haematology analyser (Sysmex Nederland, Etten-Leur, the Netherlands) according to the manufacturer's instructions.

Isolation of human PBMCs for engraftment. Human PBMCs were obtained from five healthy male volunteers (average age 26 years) under signed informed consent in accordance with the 1964 Declaration of Helsinki (revised October 2008, Seoul) and approval from the Medical Ethical Committee of the University Medical Center Groningen. Whole blood samples were obtained by venipuncture and collected in EDTA Vacutainers. PBMCs were purified by Ficoll-Paque (GE Healthcare Europe GmbH, Diegem, Belgium) density centrifugation and resuspended in Hank's buffer solution for retro-orbital injection into NSG mice at a cell dose of $5 \times 10^{6}$ cells (in $200 \mu \mathrm{l}$ ). NSG mice were reconstituted with the human PBMCs immediately after the dual injury surgical procedure. The following experimental groups were included: (i) untreated NSG mice, (ii) NSG mice given $5 \times 10^{6} \mathrm{PBMCs} 4$ weeks prior to analyses, (iii) NSG mice given $5 \times 10^{6} \mathrm{PBMCs}$ and dual arterial injury 4 weeks prior to analyses, and (iv) NSG mice which received only dual arterial injury 4 weeks prior to 
analyses. PBMCs of each donor were used to reconstitute 4 NSG mice: $n=2$ with and $n=2$ without (sham) arterial injury. Four weeks after injection, human PBMC engraftment was evaluated in spleen and peripheral blood by flowcytometry.

Antibodies and flow cytometry. Flowcytometry analysis of human PBMC cell populations in engrafted NSG mice 4 weeks after reconstitution, anti-h(uman)CD3 (clone UCHT1), hCD4 (clone RPA-T4), hCD8 (clone HIT8a), hCD14 (clone M $\varphi$ P9), hCD16 (clone 3G8), hCD45 (clone H130), and anti-m(ouse)CD45 (clone 30-F11) fluorochrome-conjugated mAbs and appropriate isotype controls (all from Biolegend, San Diego, CA, USA) were used. Antibodies were conjugated with fluorescein isothiocyanate (FITC), phycoerythrin (PE), peridinin chlorophyll (PerCP), allophycocyanin (APC), phycoerythrin-Cyanin 5 (PE-Cy5), phycoerythrin-Cyanin 7 (PE-Cy7) or Alexafluor700. Single-cell suspensions of mouse spleen (depleted of erythrocytes) and peripheral whole blood were incubated with anti-CD16/32 (BD Biosciences) for $5 \mathrm{~min}$ at $4{ }^{\circ} \mathrm{C}$ to block non-specific Fc binding. Antibodies at the appropriate dilutions were incubated with splenocytes or $200 \mu \mathrm{l}$ whole blood for $30 \mathrm{~min}$ at $4{ }^{\circ} \mathrm{C}$. Following red blood cell lysis, blood samples and labeled splenocytes cells were fixed in $1 \%$ paraformaldehyde in phosphate-buffered saline. At least 20,000 events were acquired with an LSR II instrument and analyzed using FACS DIVA software (BD Biosciences).

Measurement of human cytokine levels in NSG mice. The Cytokine Human Ultrasensitive 10-Plex Panel for the Luminex ${ }^{\circledR}$ platform (Invitrogen Corporation, Carlsbad, CA, USA) was carried out to determine the PBMC-engrafted NSG mouse plasma concentrations of human IFN $\gamma$, IL-6, IL-4, IL-1 $\beta$, IL-10, IL-8, GM-CSF, IL-2, IL-5 and TNFQ.

Quantification of neointima formation and medial area. After sacrifice, isolated zinc-fixed carotid and femoral arteries were dehydrated and embedded in paraffin. Serial cross-sections $(3 \mu \mathrm{m})$ were cut and histomorphometric analysis was performed at 20 cross-section levels (with $66 \mu \mathrm{m}$ intervals) (Fig. S1).

Sections at each level were stained with Verhoeff-van Gieson or Masson's trichrome for collagen content and slide images captured using a Hamamatsu Nanozoomer 2.0HT (Hamamatsu Photonics, Hamamatsu, Japan). Morphometric analysis was performed using Aperio Imagescope software (Aperio Technologies inc, CA, USA). Percentage intimal expansion throughout the arterial segment was calculated with the following formula: \% intimal expansion $\left.=\left(\left(A_{I}-A_{L}\right) / A_{I}\right)\right) \times 100$; where $A_{I}$ is the area within the internal elastic lamina and $A_{L}$ is the luminal area. The intima/media $(I / M)$ ratio was calculated with the following formula: $I / M$ ratio $=\left(A_{I}-A_{L}\right) /\left(A_{M}-A_{I}\right)$; where $A_{M}$ is the area within the external elastic lamina. The media and adventitial areas were also quantified. To determine loss of cells after vascular injury, the total number of cells in media and adventitia were quantified. Serial sections, as depicted in Fig. S1, were quantified to determine the mean \% intimal expansion per artery. The number of sections analyzed was an average of 12 (range 9-17) per injured artery and an average of 4 sections (range 3-5) per control artery.

Detection of human PBMC-derived cells using immunofluorescence. Zinc-fixed paraffin-embedded sections were deparaffinized in xylol, rehydrated in graded ethanol series and demi-water, and then stained using the following antibodies: anti-h- $\alpha$ SMA (clone 1A4), hCD4 (clone 4B12), hCD8 (clone C8/144B), hCD68 (clone KP-1), hCD45 (clone 2B11 + clone PD7/26), vWF (clone F8/86), hCD31 (clone JC70A) (all from DAKO, Glostrup, Denmark) and anti-mouse CD31 (clone MEC 13.3; BD Pharmingen). Sections were first blocked with 3\% BSA and subsequently incubated for 1 hour with primary antibodies. Binding of primary antibodies was detected by incubating with the appropriate fluorescently-labeled secondary antibodies: goat anti-mouse IgG1-Alexafluor488 and goat anti-mouse-Alexafluor555 (both from Invitrogen) and goat anti-rabbit IgG-Cy3 (Invitrogen) or rabbit anti-rat biotin (DAKO). Biotinylated antibody complexes were detected with streptavidin-Alexafluor555 (Invitrogen). Sections were mounted in Aqua/polymount (Polysciences Inc., Warrington, PA, USA) containing DAPI $(1.5 \mu \mathrm{g} / \mathrm{ml}$; Invitrogen). Fluorescence microscopy was performed using a Leica DMLB microscope (Leica Microsystems, Rijswijk, The Netherlands) equipped with a Leica DC300F camera and Leica QWin 2.8 software. To confirm antibody specificity, immunohistochemistry was performed on zinc-fixed (NSG spleen with or without human PBMCs) or formalin-fixed (human appendix) paraffin sections. Sections were deparaffinized in xylol, rehydrated in graded ethanol series and demi water, and subsequently stained using immunohistochemistry in a Ventana Benchmark Ultra automated IHC/ISH slide staining system (Ventana Medical Systems, Inc.). For confocal microscopy, sections were deparaffinized in xylol, rehydrated in graded ethanol series and demi water, followed by heat-induced epitope retrieval (HIER) in $10 \mathrm{mM}$ Sodium Citrate Buffer ( $\mathrm{pH}$ 6.0) for 20 min in a microwave $(300 \mathrm{~W})$. The Mouse on Mouse (M.O.M.) Basic Kit (Vector ${ }^{\circledR}$ Laboratories) was used, followed by incubation with primary antibodies (CD45/ $\alpha$ SMA and CD8/ $\alpha$ SMA) and goat anti-mouse IgG1-Alexafluor488 and goat anti-mouse-Alexafluor555 secondary antibodies as described above. Nuclei were stained with DAPI (Life Technologies) and mounted in Fluorescence Mounting Medium (DAKO). Confocal microscopy was performed using an inverted microscope (Zeiss LSM 780 NLO; Axio Observer Z1).

Phenotypic characterization of SMCs in sham-operated and injured carotid and femoral arteries. To analyse whether neointimal QSMA + cells are SMCs, immunofluorescent double labeling was performed. Heat-induced epitope retrieval (HIER) was performed in $10 \mathrm{mM}$ Sodium Citrate Buffer (pH 6.0) for 20 min in a microwave $(300 \mathrm{~W})$. Sections were blocked with $3 \%$ BSA and subsequently incubated for 1 hour with primary antibodies diluted in PBS/1\% BSA. The following primary antibodies were used: $\alpha$ SMA (mIgG2a, clone 1A4), SM22 $\alpha$ (rabbit polyclonal ab14106, Abcam), and calponin (rabbit polyclonal ab46794, Abcam). Binding of primary antibodies was detected by incubating with fluorescently-labeled secondary antibodies. For $\alpha$ SMA, 
goat-anti-mouse IgG2a-Alexafluor555 (Invitrogen) or goat-anti-mouse IgG2a-biotin (SouthernBiotech) were used. Biotin was detected with streptavidin-AlexaFluor488 conjugate (Molecular Probes). For SM22 $\alpha$ and calponin, goat anti-rabbit IgG-FITC (DAKO) or goat anti-rabbit Cy3 (Zymed Laboratories) were used. Nuclei were stained with DAPI (BioLegend) and mounted in Fluorescence Mounting Medium (DAKO). Images were acquired using TissueFAXS acquisition software (TissueGostics) on a Zeiss Axio Observer Z1 inverted microscope.

Statistical analysis. Statistical evaluations were performed with Graphpad Prism software (version 5; La Jolla, CA, USA). Data are presented as mean \pm SEM. Differences in the means of parametric data were compared by one-way ANOVA with Bonferroni post-tests. Medians of non-parametric data were compared using the Mann-Whitney $U$-test.

\section{References}

1. Jukema, J. W., Verschuren, J. J., Ahmed, T. A. \& Quax, P. H. Restenosis after PCI. Part 1: pathophysiology and risk factors. Nat. Rev. Cardiol. 9, 53-62 (2011).

2. Kearney, M. et al. Histopathology of in-stent restenosis in patients with peripheral artery disease. Circulation 95, 1998-2002 (1997).

3. Niccoli, G., Montone, R. A., Ferrante, G. \& Crea, F. The evolving role of inflammatory biomarkers in risk assessment after stent implantation. J. Am. Coll. Cardiol. 56, 1783-1793 (2010).

4. Dimayuga, P. et al. Inhibitory effect on arterial injury-induced neointimal formation by adoptive B-cell transfer in Rag-1 knockout mice. Arterioscler. Thromb. Vasc. Biol. 22, 644-649 (2002).

5. Hansson, G. K. et al. T lymphocytes inhibit the vascular response to injury. Proc. Natl. Acad. Sci. USA 88, 10530-10534 (1991).

6. Zhu, B., Reardon, C. A., Getz, G. S. \& Hui, D. Y. Both apolipoprotein E and immune deficiency exacerbate neointimal hyperplasia after vascular injury in mice. Arterioscler. Thromb. Vasc. Biol. 22, 450-455 (2002).

7. Dimayuga, P. C. et al. Enhanced neointima formation following arterial injury in immune deficient Rag-1-/- mice is attenuated by adoptive transfer of CD8 T cells. Plos One 6, e20214 (2011).

8. Dimayuga, P. C. et al. T cell modulation of intimal thickening after vascular injury: the bimodal role of IFN-gamma in immune deficiency. Arterioscler. Thromb. Vasc. Biol. 25, 2528-2534 (2005).

9. Zhou, X., Robertson, A. K., Hjerpe, C. \& Hansson, G. K. Adoptive transfer of CD4+ T cells reactive to modified low-density lipoprotein aggravates atherosclerosis. Arterioscler. Thromb. Vasc. Biol. 26, 864-870 (2006).

10. Whitman, S. C., Ravisankar, P., Elam, H. \& Daugherty, A. Exogenous interferon-gamma enhances atherosclerosis in apolipoprotein E-/- mice. Am. J. Pathol. 157, 1819-1824 (2000).

11. Tellides, G. et al. Interferon-gamma elicits arteriosclerosis in the absence of leukocytes. Nature 403, 207-211 (2000).

12. Ross, R. Atherosclerosis-an inflammatory disease. N. Engl. J. Med. 340, 115-126 (1999).

13. Iwata, H. \& Sata, M. Origin of cells that contribute to neointima growth. Circulation 117, 3060-3061 (2008).

14. Hillebrands, J. L., Klatter, F. A. \& Rozing, J. Origin of vascular smooth muscle cells and the role of circulating stem cells in transplant arteriosclerosis. Arterioscler. Thromb. Vasc. Biol. 23, 380-387 (2003).

15. Hillebrands, J. L. et al. Origin of neointimal endothelium and alpha-actin-positive smooth muscle cells in transplant arteriosclerosis. J. Clin. Invest. 107, 1411-1422 (2001).

16. Boersema, M. et al. Donor and recipient contribution to transplant vasculopathy in chronic renal transplant dysfunction. Transplantation 88, 1386-1392 (2009).

17. Caplice, N. M. et al. Smooth muscle cells in human coronary atherosclerosis can originate from cells administered at marrow transplantation. Proc. Natl. Acad. Sci. USA. 100, 4754-4759 (2003).

18. Deb, A. et al. Integrin profile and in vivo homing of human smooth muscle progenitor cells. Circulation 110, 2673-2677 (2004).

19. Simper, D., Stalboerger, P. G., Panetta, C. J., Wang, S. \& Caplice, N. M. Smooth muscle progenitor cells in human blood. Circulation 106, 1199-1204 (2002).

20. Donners, M. M. et al. The CD40-TRAF6 axis is the key regulator of the CD40/CD40L system in neointima formation and arterial remodeling. Blood 111, 4596-4604 (2008).

21. Remskar, M., Li, H., Chyu, K. Y., Shah, P. K. \& Cercek, B. Absence of CD40 signaling is associated with an increase in intimal thickening after arterial injury. Circ. Res. 88, 390-394 (2001).

22. Tanaka, K., Sata, M., Hirata, Y. \& Nagai, R. Diverse contribution of bone marrow cells to neointimal hyperplasia after mechanical vascular injuries. Circ. Res. 93, 783-790 (2003).

23. King, M. et al. A new Hu-PBL model for the study of human islet alloreactivity based on NOD-scid mice bearing a targeted mutation in the IL-2 receptor gamma chain gene. Clin. Immunol. 126, 303-314 (2008).

24. Shultz, L. D. et al. Human lymphoid and myeloid cell development in NOD/LtSz-scid IL2R gamma null mice engrafted with mobilized human hemopoietic stem cells. J. Immunol. 174, 6477-6489 (2005).

25. Ishikawa, F. et al. Development of functional human blood and immune systems in NOD/SCID/IL2 receptor \{gamma\} chain(null) mice. Blood 106, 1565-1573 (2005).

26. Song, Z. et al. CD40 is essential in the upregulation of TRAF proteins and NF-kappaB-dependent proinflammatory gene expression after arterial injury. Plos One 6, e23239 (2011).

27. Dimayuga, P. C. et al. Natural antibodies and complement modulate intimal thickening after arterial injury. Am. J. Physiol. Regul. Integr. Comp. Physiol. 297, R1593-R1600 (2009).

28. Lindner, V., Fingerle, J. \& Reidy, M. A. Mouse model of arterial injury. Circ. Res. 73, 792-796 (1993).

29. Gomez et al. Oxidative stress induces early-onset apoptosis of vascular smooth muscle cells and neointima formation in response to injury. Biosci. Rep. 35, e00227 (2015).

30. Hagensen, M. K. et al. Circulating endothelial progenitor cells do not contribute to regeneration of endothelium after murine arterial injury. Cardiovasc. Res. 93, 223-231 (2012).

31. Serruys, P. W., Regar, E. \& Carter, A. J. Rapamycin eluting stent: the onset of a new era in interventional cardiology. Heart 87, 305-307 (2002)

32. Harvey, E. J. \& Ramji, D. P. Interferon-gamma and atherosclerosis: pro- or anti-atherogenic? Cardiovasc. Res. 67, 11-20 (2005).

33. Libby, P. Inflammation in atherosclerosis. Nature 420, 868-874 (2002).

34. Zhu, S. N., Chen, M., Jongstra-Bilen, J. \& Cybulsky, M. I. GM-CSF regulates intimal cell proliferation in nascent atherosclerotic lesions. J. Exp. Med. 206, 2141-2149 (2009).

35. Kleemann, R., Zadelaar, S. \& Kooistra, T. Cytokines and atherosclerosis: a comprehensive review of studies in mice. Cardiovasc. Res. 79, 360-376 (2008)

36. Harris, A. K., Shen, J., Radford, J., Bao, S. \& Hambly, B. D. GM-CSF deficiency delays neointima formation in a normolipidemic mouse model of endoluminal endothelial damage. 87, 122-130 (2009).

37. Eefting, D. et al. The effect of interleukin-10 knock-out and overexpression on neointima formation in hypercholesterolemic APOE ${ }^{3}$-Leiden mice. Atherosclerosis 193, 335-342 (2007).

38. Mallat, Z. et al. Protective role of interleukin-10 in atherosclerosis. Circ. Res. 85, e17-e24 (1999). 
39. Mallat, Z. et al. Expression of interleukin-10 in advanced human atherosclerotic plaques: relation to inducible nitric oxide synthase expression and cell death. Arterioscler. Thromb. Vasc. Biol. 19, 611-616 (1999).

40. Feldman, L. J. et al. Interleukin-10 inhibits intimal hyperplasia after angioplasty or stent implantation in hypercholesterolemic rabbits. Circulation 101, 908-916 (2000).

41. Gibbs, V. C. et al. The extracellular domain of the human interferon gamma receptor interacts with a species-specific signal transducer. Mol Cell Biol 11, 5860-5866 (1991).

42. Shanafelt, A. B., Miyajima, A., Kitamura, T. \& Kastelein, R. A. The amino-terminal helix of GM-CSF and IL-5 governs high affinity binding to their receptors. $E M B O J$ 10, 4105-4112 (1991).

43. Fiorentino, D. F. et al. IL-10 acts on the antigen-presenting cell to inhibit cytokine production by Th1 cells. J Immunol 146, 3444-3451 (1991).

44. Hsu, D. H. et al. Expression of interleukin-10 activity by epstein-barr virus protein BCRF1. Science 250, 830-832 (1990).

45. Beckman, J. A., Creager, M. A. \& Libby, P. Diabetes and atherosclerosis: epidemiology, pathophysiology, and management. JAMA 287, 2570-2581 (2002).

46. Orasanu, G. \& Plutzky, J. The pathologic continuum of diabetic vascular disease. J. Am. Coll. Cardiol. 53, S35-S42 (2009).

47. Beckstead, J. H. A simple technique for preservation of fixation-sensitive antigens in paraffin-embedded tissues. J. Histochem. Cytochem. 42, 1127-1134 (1994).

\section{Acknowledgements}

Marian Bulthuis, Pieter Klok, Annemieke Smit-van Oosten, André Zandvoort and Michel Weij are kindly acknowledged for their technical assistance. This work was supported by the Dutch Diabetes Research Foundation (grant number 2006.01.007 to JM, JvA \& JLH). Microscopical imaging was performed at the UMCG Imaging Center (UMIC), which is supported by the Netherlands Organisation for Health Research and Development (ZonMW grant 40-00506-98-9021).

\section{Author Contributions}

J.-L.H. and J.M. conceived and designed the experiments. J.M., J.v.A., M.C.D., H.v.G. and J.-L.H. performed the experiments. D.L.G. and L.D.S. provided immunodeficient mice and contributed to data analyses. J.M. and J.v.A. analyzed the data. J.M. and J.-L.H. wrote the manuscript, which was read, commented on and agreed on by all authors prior to finalization by J.M. and J.-L.H.

\section{Additional Information}

Supplementary information accompanies this paper at http://www.nature.com/srep

Competing financial interests: The authors declare no competing financial interests.

How to cite this article: Moser, J. et al. Distinct Differences on Neointima Formation in Immunodeficient and Humanized Mice after Carotid or Femoral Arterial Injury. Sci. Rep. 6, 35387; doi: 10.1038/srep35387 (2016).

This work is licensed under a Creative Commons Attribution 4.0 International License. The images or other third party material in this article are included in the article's Creative Commons license, unless indicated otherwise in the credit line; if the material is not included under the Creative Commons license, users will need to obtain permission from the license holder to reproduce the material. To view a copy of this license, visit http://creativecommons.org/licenses/by/4.0/

(C) The Author(s) 2016 